\title{
Article \\ Determination of Colistin in Contents Derived from Gastrointestinal Tract of Feeding Treated Piglet and Broiler
}

\author{
Chun Peng ${ }^{1}$, Sanling Zuo ${ }^{1}$, Yinsheng Qiu ${ }^{1}$, Shulin Fu ${ }^{1}$ and Lijuan Peng ${ }^{2, *}$ \\ 1 School of Animal Science and Nutritional Engineering, Wuhan Polytechnic University, Changqing Garden, \\ Hankou, Wuhan 430023, China; Pengchun1998@163.com (C.P.); Zuosl30@163.com (S.Z.); \\ Qiuyinsheng6405@163.com (Y.Q.); fushulin2016@126.com (S.F.) \\ 2 School of Food Science and Engineering, Wuhan Polytechnic University, Changqing Garden, Hankou, Wuhan \\ 430023, China \\ * Correspondence: lijuan_peng@hotmail.com; Tel.: +86-278-395-6442
}

check for

updates

Citation: Peng, C.; Zuo, S.; Qiu, Y.; $\mathrm{Fu}, \mathrm{S}$;; Peng, L. Determination of Colistin in Contents Derived from Gastrointestinal Tract of Feeding Treated Piglet and Broiler. Antibiotics 2021, 10, 422. https://doi.org/ 10.3390 /antibiotics 10040422

\section{Academic Editors:}

Jesus Simal-Gandara and Carlos M. Franco

Received: 19 February 2021

Accepted: 8 April 2021

Published: 12 April 2021

Publisher's Note: MDPI stays neutral with regard to jurisdictional claims in published maps and institutional affiliations.

Copyright: (C) 2021 by the authors Licensee MDPI, Basel, Switzerland. This article is an open access article distributed under the terms and conditions of the Creative Commons Attribution (CC BY) license (https:// creativecommons.org/licenses/by/ $4.0 /)$.

\begin{abstract}
Colistin is considered as the last-resort treatment for multiantibiotic-resistant Gramnegative bacterial infections in humans. However, the oral administration of colistin to livestock and poultry results in the introduction of large amounts of colistin to the surrounding environment via urine and feces, potentially inducing the prevalence of colistin-resistant bacteria and the impact on the ecological environment. We established a quantitative mass spectrometry (MS) based method to measure colistin in contents recovered from the gastrointestinal segments of piglets and broilers, as well as colistin in feces from the animals. The mean recoveries of colistin from different matrices were between $73.2 \%$ and $103.9 \%$. The quantitation limit values for different matrices ranged from 0.37 to $1.85 \mathrm{ng} / \mathrm{g}$. In colistin-treated swine samples, the highest concentration of colistin was detected in feces samples at a level of $1248.3 \mathrm{ng} / \mathrm{g}$. However, the highest concentration of colistin in broiler samples was around $4882.9 \mathrm{ng} / \mathrm{g}$, which was found in the contents derived from broilers' ceca. The employment of the proposed method to assess colistin in animals' gastrointestinal tracts might help to understand the colistin absorption in animals' guts and the potential impact of colistin on the emergence of resistant bacteria in animals' gut flora and the ecological environment.
\end{abstract}

Keywords: colistin; swine; broiler; gastrointestinal tract; mass spectrometry

\section{Introduction}

Colistin (polymyxin E) is an old class of cationic polypeptides with broad-spectrum activity against Gram-negative bacteria by disrupting the bacterial membrane to cause cellular death $[1,2]$. In recent years, many bacterial strains have developed strong resistance to multiple antibiotics, and colistin has been considered as the last-resort treatment for the emergence of multidrug-resistant Gram-negative bacterial infections [3-5]. Colistin includes colistin A (polymyxin E1) and colistin B (polymyxin E2), which differ only in their fatty acid tails and have both been widely used as a veterinary medicine for the promotion of growth and prevention and control of diseases in livestock and poultry [6,7]. Currently, two forms of colistin are commercially available, including colistin sulfate for oral and topical use, and sodium colistimethate for parenteral and aerosol therapy. The oral administration of colistin sulfate is employed worldwide in veterinary medicine for the prevention and the treatment of colibacillosis.

However, colistin is poorly absorbed from the gastrointestinal tract [8]. A large amount of the colistin consumed by animals through feed can be excreted in urine and feces into the surrounding environment. The long-term treatment with colistin may result in the accumulation of colistin in animals' gastrointestinal tracts, and potentially induce the development and prevalence of antimicrobial-resistant bacteria and resistance genes. The tremendous increase in infections caused by antibiotic-resistant bacteria is an ecosystem problem threatening the interrelated human-animal-environment health under the "One 
Health" framework [9]. The excretion of antimicrobial-resistant bacteria and resistant genes in feces into the ecological environment may spread antibiotic resistance via crossreservoir transmission to other species or humans, by direct exposure or through the food chain and the environment. Although the prevalence of colistin resistance is currently relatively low worldwide, colistin-resistant isolates have recently been identified in several Gram-negative bacteria species, such as A. baumannii, K. pneumoniae and P. aeruginosa [10]. Extensive use of colistin in the animal industry has led to increased colistin resistance rates [11]. In 2015, the first plasmid-mediated mobile-colistin resistance ( $m c r-1)$ gene in Enterobacteriaceae was discovered in China, and subsequently found in more than 50 countries and regions $[7,12,13]$.

Lately, a positive association between the administration of colistin to swine and the accumulation of $m c r-1$ in swine manure samples was discovered [14]. Moreover, colistinresistant Escherichia coli was also observed in wild rabbits (Oryctolagus cuniculus) and wild hares (Lepus europaeus) without the treatment of colistin [15]. This suggests that colistinresistant genes might be present in the environment and cause the potential transmission of colistin-resistant E. coli between species, particularly from swine [16] or from pets [17] to humans. Based on recent studies of plasmid-mediated colistin resistance $m c r-1$ gene, livestock has been considered as the principal reservoir for colistin resistance amplification and spread [8]. Thus, colistin has been banned as a growth promoter (feed additive) in animal production in China since April 2017 [18]. The therapeutic use of colistin in livestock and poultry should be reasonably guided to reduce the prevalence and transmission of colistin resistance.

To regulate the use of colistin, respective maximum residue limits (MRLs) in animal tissues, eggs and milk have been established worldwide. For instance, MRLs of colistin in animals established in China and European communities are $50 \mathrm{ng} / \mathrm{mL}$ in milk; $150 \mathrm{ng} / \mathrm{g}$ in muscle, liver, and fat; and $200 \mathrm{ng} / \mathrm{g}$ in kidneys. To date, extensive work on the development of methods for the determination of colistin in a variety of matrices, including feed, animal tissues, eggs, milk, plasma and feces, has been performed [19-24]. However, the determination of colistin in animal tissues, eggs, milk and feces is not sufficient to validate the rationality of the therapeutic use of colistin in animals due to the poor absorption of colistin in the gastrointestinal tracts of animals after oral administration. Changes in colistin concentration in different segments of the animals' gastrointestinal tracts reflect the absorption and degradation of colistin. Therefore, studies on colistin in the gastrointestinal tracts of animals can assist in the understanding of the potential effects of colistin on the development of colistin-resistant bacteria in animals' guts and the rational therapeutic use of colistin. So far, scarce data on the concentration and distribution of colistin in animals gastrointestinal tracts after oral administration have been reported.

The goals of the study were to establish a robust and sensitive method capable in the determination of colistin in contents derived from different segments of swine and broiler gastrointestinal tracts, using ultrahigh-performance liquid chromatography-tandem mass spectrometry (UPLC-MS/MS), to investigate the concentration and absorption of colistin in different segments of animals' gastrointestinal tracts for the rational utilization of colistin.

\section{Results}

\subsection{Method Validation}

Estimates of colistin in various matrices were determined by using ion signals of selected fragment ions with a mass tolerance of $5 \mathrm{ppm}$. The corresponding chromatograms of the MS ion signals for blank and untreated samples were quite clear, and no interfering peaks were observed, demonstrating the specificity and reliability of the proposed assay (Figure 1). It was demonstrated that various matrices exhibited significant effects on ion signals for colistin. To evaluate the accuracy of the method, fortified samples were prepared for each matrix at three levels $(5,25$ and $50 \mathrm{ng} / \mathrm{g})$ by spiking the colistin standard into $2 \mathrm{~g}$ of matrix. Recoveries of colistin in different matrices ranged from $73.2 \%$ to $103.9 \%$. 
The intraday and interday measurements of colistin recoveries in different matrices were determined on three different days over a time period of one week. The within-day coefficient of variation (\%) was lower than $6.6 \%$, and between-day coefficient variation was lower than $11.4 \%$. Therefore, five matrix-fortified calibration standards set was employed to construct a linear regression equation for each matrix obtained from different segments of gastrointestinal tract of piglets and cobb broilers, to inherently correct recoveries and compensate for matrix effects. Tables 1 and 2 show the calibration curve equation, regression coefficient $\left(r^{2}\right)$, limit of detection (LOD) and limit of quantitation (LOQ) of colistin in different matrices. Good linearity was obtained with coefficients $>0.994$ for colistin in all the matrices. The LODs were in the range of $0.11 \sim 0.56 \mathrm{ng} / \mathrm{g}$ for colistin in different matrices. The LOQ for colistin in tested matrices was as low as $0.37 \mathrm{ng} / \mathrm{g}$, which was satisfactory for surveillance monitoring. As compared to the data reported previously [14], the LOQ obtained by the proposed method for colistin in swine feces was decreased by more than 10-fold.

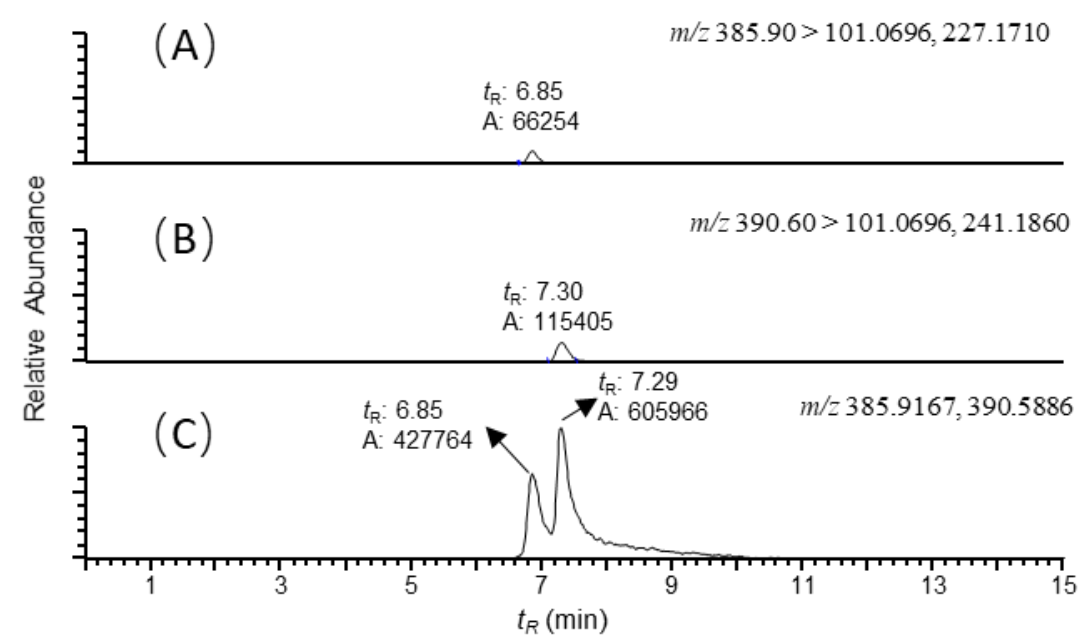

Figure 1. Reconstructed ion chromatograms of $(\mathbf{A})$ colistin $\mathrm{B}\left([\mathrm{M}+3 \mathrm{H}]^{3+}, m / z 385.90 \rightarrow 101.0696\right.$, 227.1710); $(\mathrm{B})$ colistin A $\left([\mathrm{M}+3 \mathrm{H}]^{3+}, \mathrm{m} / \mathrm{z} 390.60 \rightarrow 101.0696,241.1860\right)$; and $(\mathrm{C})$ colistin B and A $\left([\mathrm{M}+3 \mathrm{H}]^{3+}, m / z 385.9167,390.5886\right)$ recovered from the duodenum sample of swine with colistin at $5 \mathrm{ng} / \mathrm{g}$. The scales of the signals were fixed to the maximum response of the analytes. Mass tolerance was set at 5 ppm.

Table 1. Calibration curve equation, $r^{2}$, LOD and LOQ values for colistin in each matrix from the swine gastrointestinal tracts and feces.

\begin{tabular}{ccccc}
\hline Gastrointestinal Segment/Feces & Linear Equation & $\boldsymbol{r}^{2}$ Values & LOD (ng/g) & LOQ (ng/g) \\
\hline Stomach & $\mathrm{y}=39926 \mathrm{x}-168707$ & 0.9992 & 0.28 & 0.93 \\
Duodenum & $\mathrm{y}=50445 \mathrm{x}-521015$ & 0.9968 & 0.13 & 0.96 \\
Jejunum & $\mathrm{y}=44455 \mathrm{x}-114407$ & 0.9974 & 0.13 & 0.45 \\
Ileum & $\mathrm{y}=42583 \mathrm{x}-234343$ & 0.9964 & 0.32 & 0.42 \\
Cecum & $\mathrm{y}=40806 \mathrm{x}-160046$ & 0.9948 & 0.98 & 0.93 \\
Feces & $\mathrm{y}=51895 \mathrm{x}-307464$ & 0.9997 & & 0.08 \\
\hline
\end{tabular}

Table 2. Calibration curve equation, $r^{2}$, LOD and LOQ values for colistin in each matrix from the broiler gastrointestinal tracts and feces.

\begin{tabular}{ccccc}
\hline Gastrointestinal Segment/Feces & Linear Equation & $r^{2}$ Values & LOD (ng/g) & LOQ (ng/g) \\
\hline Gizzard & $\mathrm{y}=35126 \mathrm{x}+539167$ & 0.9990 & 0.26 & 0.86 \\
Proventriculus & $\mathrm{y}=41242 \mathrm{x}-87577$ & 0.9965 & 0.17 & 0.57 \\
Duodenum & $\mathrm{y}=41551 \mathrm{x}+215899$ & 0.9962 & 0.11 & 0.37 \\
Jejunum & $\mathrm{y}=38034 \mathrm{x}+134109$ & 0.9958 & 0.42 & 1.39 \\
Ileum & $\mathrm{y}=38529 \mathrm{x}-155306$ & 0.9944 & 0.27 & 0.89 \\
Cecum & $\mathrm{y}=42291 \mathrm{x}+260184$ & 0.9970 & 0.23 & 0.78 \\
Feces & $\mathrm{y}=42184 \mathrm{x}+231232$ & 0.9966 & 0.56 & 1.85 \\
\hline
\end{tabular}




\subsection{Analysis of Swine Samples}

Colistin was found in all samples from colistin-treated piglets, but no colistin was detected in any untreated swine sample. The concentrations of colistin recovered from the different segments of the swine gastrointestinal tracts and feces are reported in Figure 2. Among the tested samples, the average concentration of colistin in feces samples was approximately $1248.3 \mathrm{ng} / \mathrm{g}$, which was statistically higher than that in the content of the gastrointestinal tracts. The detected concentrations of colistin in swine feces samples were consistent with earlier findings in which the concentration of colistin in fresh manure samples collected from five swine farms was reported in the range of below the LOQ to $17,383 \mathrm{ng} / \mathrm{g}$ [14]. For content samples from the gastrointestinal tract, the content from the cecum contained relatively high concentrations of colistin at a level of $398.3 \pm 161.2 \mathrm{ng} / \mathrm{g}$ (mean \pm standard deviation). Taking moisture into account, the average amounts of colistin in feces and in content recovered from the cecum were comparably high $(\sim 3300 \mathrm{ng} / \mathrm{g})$ when calculations were based on the dried weight of the samples. Compared to the samples mentioned above, samples recovered from the stomach contained a relatively lower level of colistin, followed by samples from the duodenum and jejunum, which contained similar concentrations of colistin at an average level of $\sim 30 \mathrm{ng} / \mathrm{g}$. Data for the ileum sample were not obtained due to insufficient contents for analysis. The trend of colistin distribution in the swine gastrointestinal tracts could be due to the absorption of colistin in swine duodenum and jejunum and the accumulation of colistin in swine ceca.

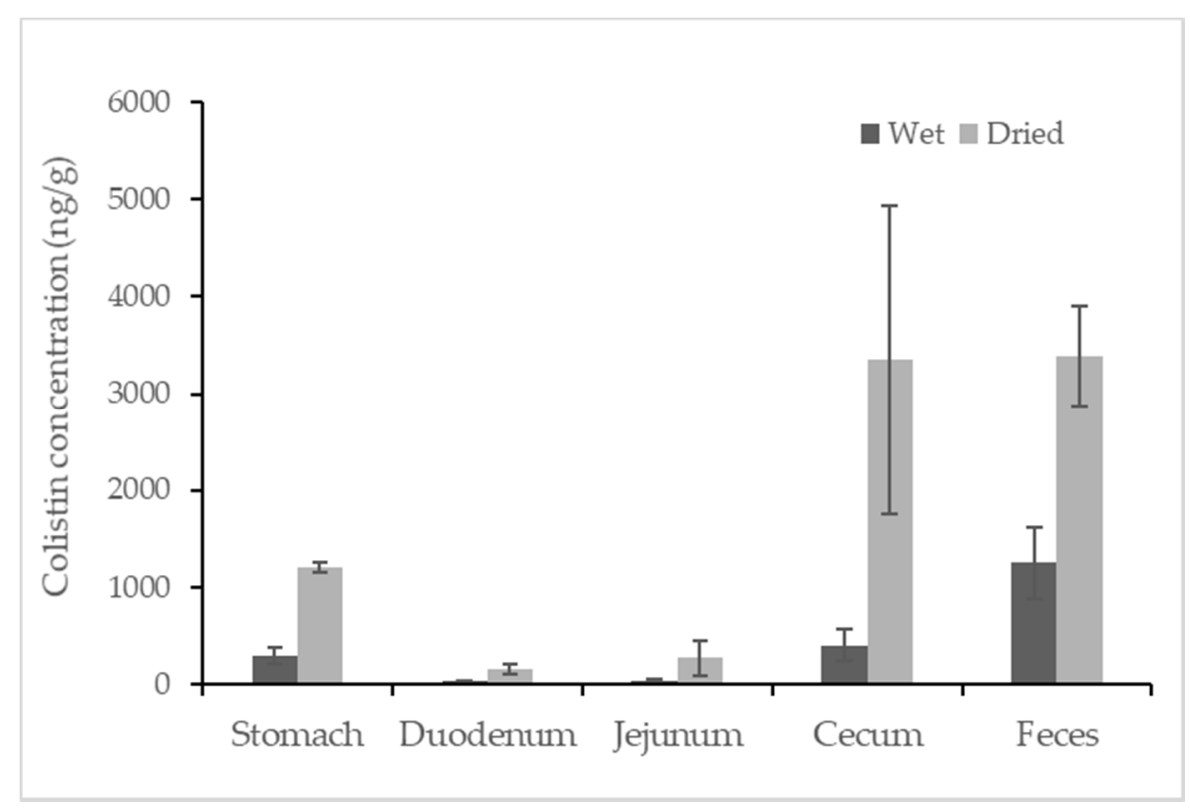

Figure 2. Concentration of colistin in contents recovered from segments of the swine gastrointestinal tracts and feces. The calculation was based on both wet weight (black) and dried weight (grey) of each content $(n=4)$.

\subsection{Analysis of Broiler Samples}

No colistin was detected in untreated broiler samples. All samples collected from colistin-treated broilers contained colistin, with the highest concentration observed in content recovered from the cecum (Figure 3). Colistin determined in content samples from the cecum ranged from 1348.0 to $11528.6 \mathrm{ng} / \mathrm{g}$, with a high coefficient of variance at $62.9 \%$, indicating significant individual variation among broilers. For content samples recovered from different segments of the broilers' gastrointestinal tracts, the average concentrations of colistin were 1145.7, 448.3, 383.7, 1572.3, 2056.1 and $4882.9 \mathrm{ng} / \mathrm{g}$ for gizzard, proventriculus, duodenum, jejunum, ileum and cecum samples, respectively. 


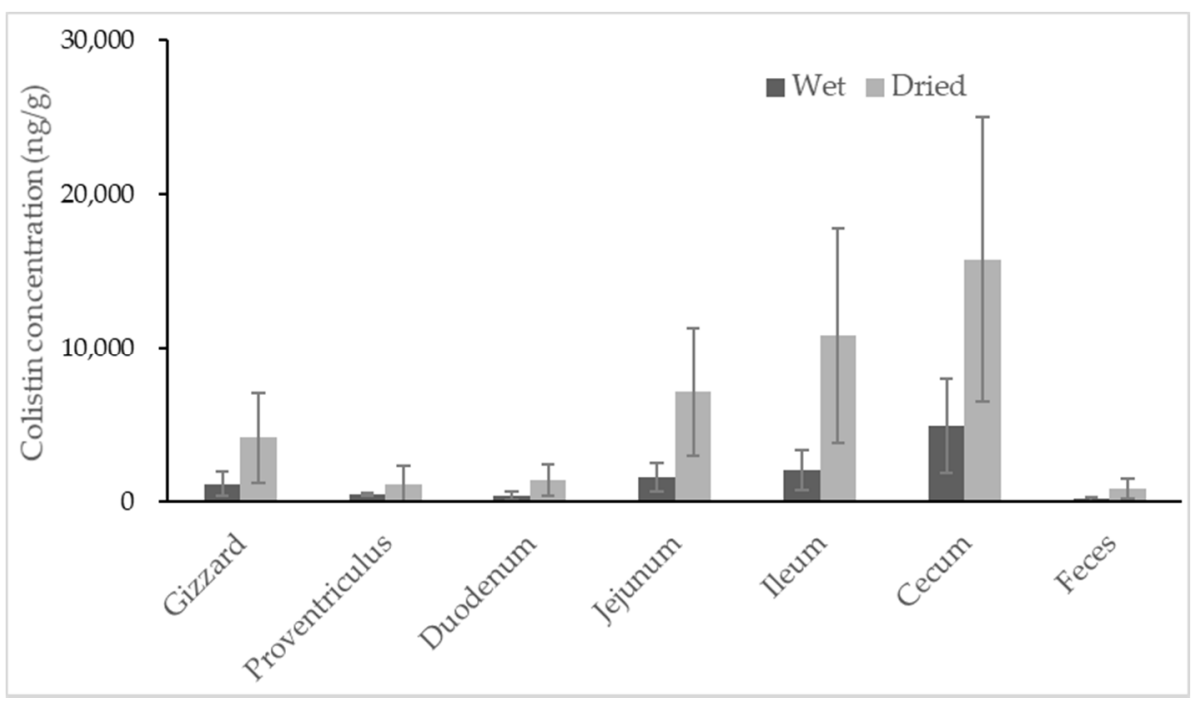

Figure 3. Concentration of colistin in contents recovered from segments of the broiler gastrointestinal tracts and feces. The calculation was based on both wet weight (black) and dried weight (grey) of each content $(n=8)$.

\section{Discussion}

The compositions of contents recovered from different segments of animals' gastrointestinal tracts and feces are distinct from one another. The matrix effect usually has a significant influence on the recovery of analytes. Moreover, the amount of colistin in different matrices derived from the gastrointestinal tracts of colistin-treated animals may vary considerably due to the absorption and degradation of colistin. Thus, the primary challenges for establishing a universal method to determine colistin in these matrices are appropriate recoveries and sensitivity. In this study, the solid phase extraction (SPE) purification of colistin in different matrices by a weak cation exchange (WCX) cartridge, followed by vacuum centrifugation for dryness, ensured the appropriate recoveries of colistin, especially when analytes were at low concentrations. The employment of high-resolution mass spectrometry combined with ultra-high performance liquid chromatography under optimized conditions offered lower LOQs for colistin in the different analyzed matrices. The LOQ values for colistin in tested matrices ranged from 0.37 to $1.85 \mathrm{ng} / \mathrm{g}$, which were far below the MRLs for the animals' tissues. This suggests that the presented assay can help to monitor the absorption and degradation of colistin in animals' gastrointestinal tracts.

It is well known that ion suppression or ion enhancement caused by the sample matrix and interferences from metabolites is always the main issue in the effect of the accuracy and reliability of UPLC-MS/MS-based assays for biological samples [25]. The colistin A and $\mathrm{B}$ examined are cationic polypeptides, which generally form multicharged ions under electrospray ionization (ESI). In this study, both colistin A and colistin B appeared as triple and quadruple pronated ions in mass spectra at a $\mathrm{m} / \mathrm{z}$ range $<400$, arising with severe interference ions from the matrix, solvent and other contaminants (Figure 4). Quantitative analysis of colistin in different matrices was based on the ion signals of fragment ions of colistin A and B, ensuring the specificity and reliability of the method. However, the fragmentation of parent ions usually leads to decreased ion signals, thus affecting the sensitivity of the method. The extraction of parent ions from full scan mass spectra with a mass tolerance of $5 \mathrm{ppm}$ could eliminate most interference ions (Figure 1C). According to our data, selecting the ion signal of parent ions instead of fragment ions for quantitation could lower the LOQ for each matrix by at least 3-fold. Moreover, lower LOQs may be achieved by assaying more samples, or by the selective enrichment of colistin prior to MS/MS analysis. 


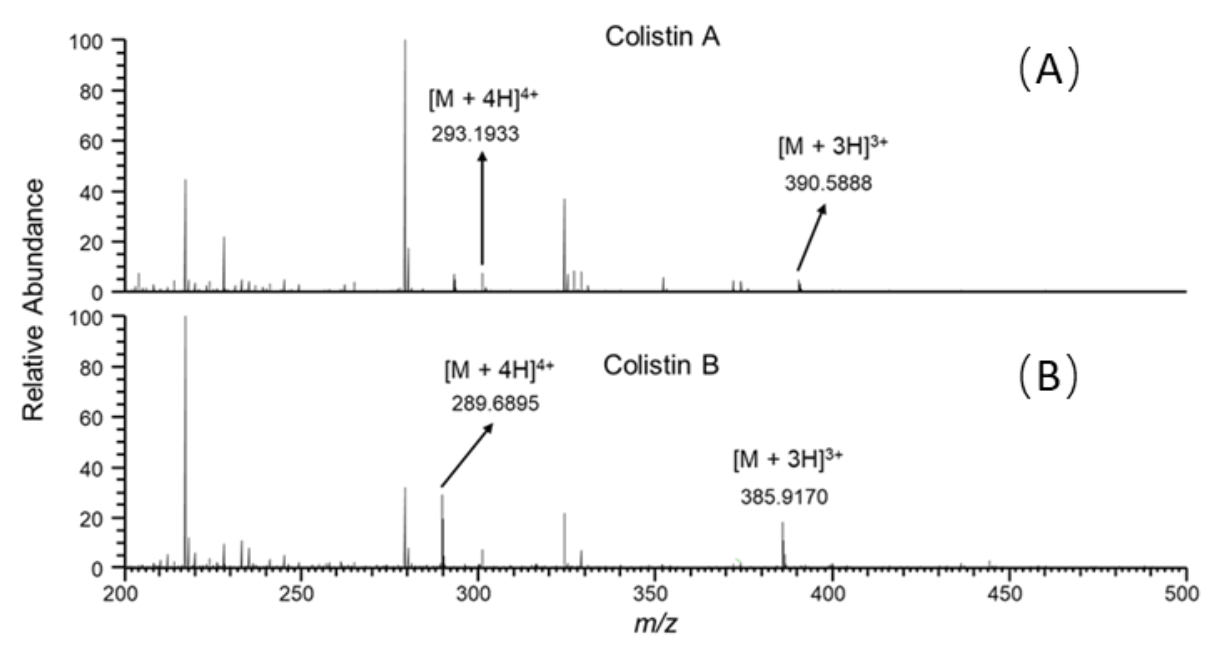

Figure 4. Full scan mass spectra of the duodenum sample from swine with colistin at $5 \mathrm{ng} / \mathrm{g}$, acquired at retention time of (A) 7.30 and (B) $6.85 \mathrm{~min}$.

Nutrients and drugs are known to be mainly absorbed in the small intestine of animals and humans after oral administration. The dosing regimen of colistin in clinical use depends upon the pharmacokinetics and pharmacodynamics of colistin in humans [26]. Meanwhile, the absorption of oral drugs in the digestive tract is crucial for the dosing regimen as well. Unlike for human medicine, a few studies have been conducted in animals to evaluate the pharmacokinetics and pharmacodynamics of colistin following oral administration $[8,27,28]$. One study has measured colistin in the whole intestinal tracts (from the duodenum to ileum) of pigs after a single oral administration, using highperformance liquid chromatography assays (HPLC-Fluorescence). Since colistin cannot be readily detected by HPLC detectors, the derivatization of colistin was conducted prior to HPLC analysis. Peak concentrations of colistin in samples from the duodenum to ileum were achieved rapidly after administration, at the highest level of $\sim 90,000 \mathrm{ng} / \mathrm{g}$. However, no colistin was detected in any of the samples from duodenum to ileum after $4 \mathrm{~h}$ [28]. According to our data, this was likely due to the poor sensitivity of the HPLC assay. Our study provided the trend of colistin distribution in the swine gastrointestinal tract, indicating that the absorption of colistin probably occurred in the swine duodenum and jejunum. The slightly higher amount of colistin found in contents recovered from the swine jejunum might be attributed to less absorption of colistin in the jejunum than in the duodenum of swine. The accumulation and lower absorption of colistin might contribute to significantly increased amounts of colistin in the contents of swine ceca. As polypeptides, colistin may be degraded by proteolytic enzymes and microorganisms in the swine gastrointestinal tract. Differences in the cecal microbiota of individuals reportedly occur in swine [29], suggesting that the degradation of colistin in the swine cecum may differ in individuals. This may explain the observed high variability of colistin in the cecum samples of swine.

The two lowest concentrations of colistin were discovered in content samples from a broiler's proventriculus and duodenum, which were probably the primary location of colistin absorption. The average concentrations of colistin gradually increased in the content of the jejunum, ileum and cecum, suggesting less or no absorption of colistin in these areas. It was found that colistin recovered from all segments of the gastrointestinal tract was highly varied in individuals. The specific dominant bacterial community was reported in every broiler, as well as every compartment of each broiler's intestinal tract [30,31]. Thus, the degradation of colistin caused by bacteria in each segment of broiler intestinal tract may be different from one another, resulting in high variabilities of colistin recovered from each segment of intestinal tract among individuals. It is noteworthy that the feces samples of broilers had very little colistin, with an average concentration of $147.7 \mathrm{ng} / \mathrm{g}$. Regardless of the moisture, the estimates of colistin in samples from the cecum and feces (mean \pm stan- 
dard deviation) were $15766.0 \pm 9249.2$ and $823.4 \pm 616.4 \mathrm{ng} / \mathrm{g}$, respectively. The amount of colistin significantly decreased from the cecum to feces by at least 5-fold. The rectum of broilers is short; colistin can usually be excreted quickly from the broiler's cecum to the surrounding environment. The disappearance of most colistin during transferring from the cecum to feces could not be caused by the absorption of colistin in the large intestine of broilers, but possibly by the degradation of colistin in the large intestine. Unfortunately, the concentration of colistin in content samples from the large intestine was unavailable due to insufficient samples for analysis. Studies are ongoing to reveal the potential metabolisms of colistin in broilers' large intestines.

The presented data signify that colistin used in veterinary medicine by oral administration leads to elevated colistin in the contents of animals' gastrointestinal tracts and feces, and thereby explains the importance and urgency of the judicious therapeutic use of colistin in animals.

\section{Materials and Methods}

\subsection{Chemicals and Materials}

Colistin sulfate (colistin A and colistin B mixture) was purchased from the National Institute of Control of Pharmaceutical and Biological Products (Beijing, China). LC-MS grade methanol, acetonitrile, water and formic acid (50\%) were obtained from Thermo Fisher Scientific (San Jose, CA, USA). Oasis WCX SPE cartridges (3 cc/60 mg) were from Waters (Milford, MA, USA). All other reagents were analytical grade and purchased from Sinopharm Chemical Reagent (Shanghai, China).

\subsection{Animal Treatment}

Eight weaned piglets (Duroc $\times$ Landrace $\times$ Large white) 3 4 weeks of age were used in a 14-day feeding trial. First, all piglets were fed with the base diet (Table S1) for an adapting period of 6 days. Subsequently, four piglets fed the base diet were kept as untreated controls, and the other four piglets were fed the base diet, which was supplemented with colistin sulphate at $20 \mathrm{mg} / \mathrm{kg}$ of body weight for 14 consecutive days. Thereafter, feces were collected, and the piglets were slaughtered to obtain contents from the swine gastrointestinal segments, including stomach, duodenum, jejunum and cecum. The sixteen cobb broilers 1-3 days in age were selected and fed with a drug-free balanced diet (Table S2) during the 14-day adaption period and the subsequent treatment period. Then, eight broilers were kept as untreated controls, and the other eight broilers were fed with colistin at $20 \mathrm{mg} / \mathrm{kg}$ of body weight for 14 consecutive days. At the end of the trial, treated and untreated animals were sacrificed, and the contents derived from the gizzard, proventriculus, duodenum, jejunum, ileum and cecum were recovered. All samples were stored at $-80^{\circ} \mathrm{C}$ before being analyzed.

\subsection{Extraction of Colistin from Contents and Feces}

The extraction procedure was based on the methods developed by $\mathrm{Fu}$ [20] with some modifications. Briefly, $2 \mathrm{~g}$ of samples was lyophilized to dryness and extracted twice with $10 \mathrm{~mL}$ of $10 \%$ trichloroacetic acid: acetonitrile $(40: 60, v / v)$, and the combined extraction solution was adjusted to $\mathrm{pH}$ 9.0. Thereafter, the extraction solution was applied to Oasis WCX SPE cartridges which were prewashed with $3 \mathrm{~mL}$ of methanol and $3 \mathrm{~mL}$ of water. The cartridges were then rinsed with $3 \mathrm{~mL}$ of water, $3 \mathrm{~mL}$ of methanol and $3 \mathrm{~mL}$ of $5 \%$ formic acid in water. Eventually, the colistin was eluted with $5 \mathrm{~mL}$ of $20 \%$ formic acid in methanol. The eluent was evaporated to dryness by vacuum centrifugation at $50{ }^{\circ} \mathrm{C}$ and resuspended in $500 \mu \mathrm{L}$ of deionized water prior to measurement using an UPLC-MS/MS system. 


\subsection{UPLC-MS/MS}

Chromatography of colistin was conducted with an UltiMate 3000 UPLC system (Thermo Fisher, San Jose, CA, USA) equipped with an Acquity UPLC BEH C18 column $(2.1 \mathrm{~mm} \times 100 \mathrm{~mm} ; 1.7 \mu \mathrm{m}$ particle size; Waters, Milford, MA, USA). The mobile phase A was $0.2 \%$ formic acid, $5 \%$ acetonitrile in $\mathrm{H}_{2} \mathrm{O}$; B was $100 \%$ acetonitrile. The colistin was resolved with a linear gradient starting from $95 \%$ A solvent to $70 \%$ B solvent over $5 \mathrm{~min}$ and then to $5 \% \mathrm{~A}$ at $10 \mathrm{~min}$, at a flow rate of $200 \mu \mathrm{L} / \mathrm{min}$.

MS spectra were acquired at positive ionization mode with a hybrid quadrupoleobitrap mass spectrometer (Q Exactive, Thermo Fisher, San Jose, CA, USA). The sheath gas flow rate was $25 \mathrm{~mL} / \mathrm{min}$; the auxiliary gas flow rate was $15 \mathrm{~mL} / \mathrm{min}$; the spray voltage was $3.2 \mathrm{kV}$; and the temperature of the capillary tube was set to $320^{\circ} \mathrm{C}$. One microscan was used for data acquisition. The resolution settings were full MS target 70,000 and MS/MS target 17,500; normalized CE $20 \%$ and $22 \%$ were employed for colistin A and colistin $\mathrm{B}$, respectively. Data manipulations were employed using Xcalibur version 4.0 software. Quantitative analyses were conducted in the selective reaction monitoring (SRM) scan mode. The two most abundant fragment ions were selected from each analyte for analysis (Figure S1). The transitions monitored and approximate chromatographic retention times $\left(t_{\mathrm{R}}\right)$ were as follows: colistin $\mathrm{A}\left([\mathrm{M}+3 \mathrm{H}]^{3+}\right), \mathrm{m} / z 390.60>101.0696,241.1860, t_{R}=7.30 \mathrm{~min}$; colistin $\mathrm{B}\left([\mathrm{M}+3 \mathrm{H}]^{3+}\right), \mathrm{m} / z 385.90>101.0696,227.1710, t_{R}=6.85 \mathrm{~min}$. The mass tolerance was set to $5 \mathrm{ppm}$ for fragment ions.

\subsection{Method Validation}

Estimates of colistin were determined by the total signals of selected fragment ions for colistin A and B. The method was validated in terms of specificity, recovery, accuracy, precision and linearity. The specificity of the method was investigated by checking potential ion signals of interferences from blank and untreated animal samples. The accuracy and recovery of the method was evaluated by recovery experiments at 3 fortified concentrations of colistin $(5,25,50 \mathrm{ng} / \mathrm{g})$ in contents derived from different segments of gastrointestinal tract of piglets and broilers. Samples were prepared in triplicate. The precision was determined by intraday and interday assay. The LOD and LOQ values of colistin in different matrices were calculated by the signal-to-noise ratio of $3(\mathrm{~S} / \mathrm{N}=3)$ and $10(\mathrm{~S} / \mathrm{N}=10)$, respectively.

\subsection{UPLC-MS/MS Analysis for Real Samples}

Colistin-treated samples and controls were subject to analysis using the proposed method, including stomach, duodenum, jejunum and cecum content samples and feces samples recovered from 4 treated and 4 untreated piglets; gizzard, proventriculus, duodenum, jejunum, ileum and cecum content samples and feces samples from 8 treated and 8 control cobb broilers. Five-point matrix-matched calibration standard curves, over a concentration range covering the concentrations expected in animal samples, were prepared with each content sample from the gastrointestinal segments and feces samples recovered from untreated animals. Confirmation criteria were that the selected fragment ions in the samples were within the tolerance range of $5 \mathrm{ppm}$ in matrix-matched standards, and the retention time of the compound in the samples within $\pm 2 \%$ of the retention time of the matrix-matched standards.

\section{Conclusions}

Currently, the oral administration of colistin is still among the main means for the treatment of infections caused by multidrug resistance in animal husbandry. A large amount of colistin residue and potential colistin-resistant bacteria can be excreted into the surrounding environment via animals' feces, which affects environmental microbes, causing an impact on the prevalence of colistin resistance in humans. However, extensive work has focused on the pharmacokinetics and pharmacodynamics of colistin, while scarce research has been carried out on the absorption of colistin in animals' gastrointestinal tracts. 
This is the first time that an MS-based assay has been developed to measure colistin in contents obtained from different segments of gastrointestinal tract of swine and broilers. The proposed method is demonstrated to be accurate and robust. The LOQs of colistin in the matrices tested were as low as $0.37 \mathrm{ng} / \mathrm{g}$. Colistin was detected in all colistin-treated samples. For possible samples with a concentration below LODs, lower LOQs may be achieved by assaying more samples, or by the selective enrichment of colistin prior to MS/MS analysis. The employment of the proposed method to assess the concentration of colistin in contents recovered from different segments of animals' gastrointestinal tracts will help to understand the colistin absorption in animals' guts, and the potential impact on animals' gut flora and the ecological environment. Furthermore, the validated method may assist in monitoring colistin in the animal treatment to ensure a judicious therapeutic use of colistin in animals.

Supplementary Materials: The following are available online at https:/ /www.mdpi.com/article/10 .3390/antibiotics10040422/s1, Table S1: Diet composition for swine as fed; Table S2: Diet composition for broiler as fed; Figure S1: Product ion mass spectra of $(\mathrm{A})$ colistin $\mathrm{B}\left([\mathrm{M}+3 \mathrm{H}]^{3+} \mathrm{at} \mathrm{m} / \mathrm{z} 385.90\right)$ and (B) colistin $\mathrm{A}\left([\mathrm{M}+3 \mathrm{H}]^{3+}\right.$ at $\left.\mathrm{m} / \mathrm{z} 390.60\right)$.

Author Contributions: Conceptualization, Y.Q. and L.P.; methodology, Y.Q. and L.P.; validation, C.P., S.Z. and S.F.; formal analysis, C.P. and S.Z.; investigation, L.P.; resources, Y.Q.; data curation, C.P. and L.P.; writing—original draft preparation, L.P.; writing—review and editing, Y.Q. and L.P.; visualization, L.P.; supervision, Y.Q.; project administration and funding acquisition, Y.Q. All authors have read and agreed to the published version of the manuscript.

Funding: This research was funded by the National Key R\&D Plan (grant no.2018YFD0500301).

Institutional Review Board Statement: This study was conducted in strict accordance with China Regulations for the Administration of Affairs Concerning Experimental Animals 1988 and the Hubei Regulations for the Administration of Affairs Concerning Experimental Animals 2005. The animal experiments and animal care were approved by the Animal Care and Use Committee of Wuhan Polytechnic University, Hubei Province, China (EM950, 5 November 2020).

Data Availability Statement: Data is contained within the article.

Conflicts of Interest: The authors declare no conflict of interest.

\section{References}

1. Li, J.; Nation, R.L.; Milne, R.W.; Turnidge, J.D.; Coulthard, K. Evaluation of colistin as an agent against multi-resistant Gramnegative bacteria. Int. J. Antimicrob. Agents 2005, 25, 11-25. [CrossRef]

2. Moffatt, J.H.; Harper, M.; Harrison, P.; Hale, J.D.; Vinogradov, E.; Seemann, T.; Henry, R.; Crane, B.; St Michael, F.; Cox, A.D.; et al. Colistin resistance in Acinetobacter baumannii is mediated by complete loss of lipopolysaccharide production. Antimicrob. Agents Chemother. 2010, 54, 4971-4977. [CrossRef]

3. Spapen, H.; Jacobs, R.; Van Gorp, V.; Troubleyn, J.; Honoré, P.M. Renal and neurological side effects of colistin in patients-2011. Ann. Intensive Care 2011, 1, 14. [CrossRef]

4. Bialvaei, A.Z.; Samadi Kafil, H. Colistin, mechanisms and prevalence of resistance. Curr. Med. Res. Opin. 2015, 31, 707-721. [CrossRef]

5. Andrade, F.F.; Silva, D.; Rodrigues, A.; Pina-Vaz, C. Colistin Update on Its Mechanism of Action and Resistance, Present and Future Challenges. Microorganisms 2020, 8, 1716. [CrossRef] [PubMed]

6. Rhouma, M.; Beaudry, F.; Letellier, A. Resistance to colistin: What is the fate for this antibiotic in pig production? Int. J. Antimicrob. Agents 2016, 48, 119-126. [CrossRef] [PubMed]

7. Vounba, P.; Rhouma, M.; Arsenault, J.; Bada Alambédji, R.; Fravalo, P.; Fairbrother, J.M. Prevalence of colistin resistance and mcr-1/mcr-2 genes in extended-spectrum $\beta$-lactamase/AmpC-producing Escherichia coli isolated from chickens in Canada, Senegal and Vietnam. J. Glob. Antimicrob. Resist. 2019, 19, 222-227. [CrossRef] [PubMed]

8. Rhouma, M.; Beaudry, F.; Thériault, W.; Letellier, A. Colistin in Pig Production: Chemistry, Mechanism of Antibacterial Action, Microbial Resistance Emergence, and One Health Perspectives. Front. Microbiol. 2016, 7, 1789. [CrossRef]

9. Puvača, N.; de Llanos Frutos, R. Antimicrobial Resistance in Escherichia coli Strains Isolated from Humans and Pet Animals. Antibiotics 2021, 10, 69. [CrossRef] [PubMed]

10. Park, Y.K.; Choi, J.Y.; Shin, D.; Ko, K.S. Correlation between overexpression and amino acid substitution of the PmrAB locus and colistin resistance in Acinetobacter baumannii. Int. J. Antimicrob. Agents 2011, 37, 525-530. [CrossRef] 
11. Ngogang, M.P.; Ernest, T.; Kariuki, J.; Mouliom Mouiche, M.M.; Ngogang, J.; Wade, A.; van der Sande, M.A.B. Microbial Contamination of Chicken Litter Manure and Antimicrobial Resistance Threat in an Urban Area Setting in Cameroon. Antibiotics 2020, 10, 20. [CrossRef]

12. Liu, Y.-Y.; Wang, Y.; Walsh, T.R.; Yi, L.-X.; Zhang, R.; Spencer, J.; Doi, Y.; Tian, G.; Dong, B.; Huang, X.; et al. Emergence of plasmid-mediated colistin resistance mechanism MCR-1 in animals and human beings in China: A microbiological and molecular biological study. Lancet Infect. Dis. 2016, 16, 161-168. [CrossRef]

13. Mobasseri, G.; Teh, C.S.J.; Ooi, P.T.; Thong, K.L. The emergence of colistin-resistant Klebsiella pneumoniae strains from swine in Malaysia. J. Glob. Antimicrob. Resist. 2019, 17, 227-232. [CrossRef] [PubMed]

14. Xia, X.; Wang, Z.; Fu, Y.; Du, X.-D.; Gao, B.; Zhou, Y.; He, J.; Wang, Y.; Shen, J.; Jiang, H.; et al. Association of colistin residues and manure treatment with the abundance of mcr-1 gene in swine feedlots. Environ. Int. 2019, 127, 361-370. [CrossRef] [PubMed]

15. Dotto, G.; Giacomelli, M.; Grilli, G.; Ferrazzi, V.; Carattoli, A.; Fortini, D.; Piccirillo, A. High prevalence of oqxAB in Escherichia coli isolates from domestic and wild lagomorphs in Italy. Microb. Drug Resist. 2014, 20, 118-123. [CrossRef]

16. Olaitan, A.O.; Thongmalayvong, B.; Akkhavong, K.; Somphavong, S.; Paboriboune, P.; Khounsy, S.; Morand, S.; Rolain, J.-M. Clonal transmission of a colistin-resistantEscherichia colifrom a domesticated pig to a human in Laos: Table 1. J. Antimicrob. Chemother. 2015, 70, 3402-3404. [CrossRef]

17. Zhang, X.-F.; Doi, Y.; Huang, X.; Li, H.-Y.; Zhong, L.-L.; Zeng, K.-J.; Zhang, Y.-F.; Patil, S.; Tian, G.-B. Possible Transmission ofmcr-1-HarboringEscherichia colibetween Companion Animals and Human. Emerg. Infect. Dis. 2016, 22, $1679-1681$. [CrossRef] [PubMed]

18. Walsh, T.R.; Wu, Y. China bans colistin as a feed additive for animals. Lancet Infect. Dis. 2016, 16, 1102-1103. [CrossRef]

19. Kaufmann, A.; Widmer, M. Quantitative analysis of polypeptide antibiotic residues in a variety of food matrices by liquid chromatography coupled to tandem mass spectrometry. Anal. Chim. Acta 2013, 797, 81-88. [CrossRef] [PubMed]

20. Fu, Q.; Li, X.; Zheng, K.; Ke, Y.; Wang, Y.; Wang, L.; Yu, F.; Xia, X. Determination of colistin in animal tissues, egg, milk, and feed by ultra-high performance liquid chromatography-tandem mass spectrometry. Food Chem. 2018, 248, 166-172. [CrossRef]

21. Cangemi, G.; Barco, S.; Castagnola, E.; Tripodi, G.; Favata, F.; D'Avolio, A. Development and validation of UHPLC-MS/MS methods for the quantification of colistin in plasma and dried plasma spots. J. Pharm. Biomed. Anal. 2016, 129, 551-557. [CrossRef] [PubMed]

22. Choosakoonkriang, S.; Supaluknari, S.; Puangkaew, P. High Performance Liquid Chromatographic Method for Determination of Colistin Sulfate and its Application in Medicated Premixand Animal Feed. Int. Sch. Sci. Res. Innov. 2013, 7. [CrossRef]

23. Boison, J.O.; Lee, S.; Matus, J. A multi-residue method for the determination of seven polypeptide drug residues in chicken muscle tissues by LC-MS/MS. Anal. Bioanal. Chem. 2015, 407, 4065-4078. [CrossRef] [PubMed]

24. Dotsikas, Y.; Markopoulou, C.K.; Koundourellis, J.E.; Loukas, Y.L. Validation of a novel LC-MS/MS method for the quantitation of colistin A and B in human plasma. J. Sep. Sci. 2011, 34, 37-45. [CrossRef] [PubMed]

25. Matuszewski, B.K.; Constanzer, M.L.; Chavez-Eng, C.M. Strategies for the Assessment of Matrix Effect in bioanalytical methods2003. Anal. Chem. 2003, 75, 12.

26. Gregoire, N.; Aranzana-Climent, V.; Magreault, S.; Marchand, S.; Couet, W. Clinical Pharmacokinetics and Pharmacodynamics of Colistin. Clin. Pharm. 2017, 56, 1441-1460. [CrossRef] [PubMed]

27. Tang, S.S.; Gong, L.J.; He, J.K.; Jin, X.; Xiao, X.L. Residue depletion of colistin in swine after intramuscular administration. J. S. Afr. Vet. Assoc. 2009, 80, 41-44. [CrossRef]

28. Guyonnet, J.; Manco, B.; Baduel, L.; Kaltsatos, V.; Aliabadi, M.H.; Lees, P. Determination of a dosage regimen of colistin by pharmacokinetic/pharmacodynamic integration and modeling for treatment of G.I.T. disease in pigs. Res. Vet. Sci. 2010, 88, 307-314. [CrossRef]

29. Tan, Z.; Yang, T.; Wang, Y.; Xing, K.; Zhang, F.; Zhao, X.; Ao, H.; Chen, S.; Liu, J.; Wang, C. Metagenomic Analysis of Cecal Microbiome Identified Microbiota and Functional Capacities Associated with Feed Efficiency in Landrace Finishing Pigs. Front. Microbiol. 2017, 8, 1546. [CrossRef]

30. Sergeant, M.J.; Constantinidou, C.; Cogan, T.A.; Bedford, M.R.; Penn, C.W.; Pallen, M.J. Extensive microbial and functional diversity within the chicken cecal microbiome. PLoS ONE 2014, 9, e91941. [CrossRef]

31. van der Wielen, P.W.; Keuzenkamp, D.A.; Lipman, L.J.; van Knapen, F.; Biesterveld, S. Spatial and temporal variation of the intestinal bacterial community in commercially raised broiler chickens during growth. Microb. Ecol. 2002, 44, 286-293. [CrossRef] [PubMed] 